Copyright by the American Physical Society. Wohlgenannt, M ; Graupner, W ; Leising, G ; et al., Aug 15, 1999. "Photogeneration and recombination processes of neutral and charged excitations in films of a ladder-type poly(para-phenylene)," PHYSICAL REVIEW B 60(8): 5321-5330. DOI: 10.1103/PhysRevB.60.5321.

\title{
Photogeneration and recombination processes of neutral and charged excitations in films of a ladder-type poly(para-phenylene)
}

\author{
M. Wohlgenannt \\ Department of Physics, University of Utah, Salt Lake City, Utah 84112 \\ and Institut für Festkörperphysik, Technische Universität Graz, Petersgasse 16, A-8010 Graz, Austria \\ W. Graupner \\ Department of Physics, Virginia Tech, Blacksburg, Virginia 24061-0435 \\ and Institut für Festkörperphysik, Technische Universität Graz, Petersgasse 16, A-8010 Graz, Austria \\ G. Leising \\ Institut für Festkörperphysik, Technische Universität Graz, Petersgasse 16, A-8010 Graz, Austria \\ Z. V. Vardeny \\ Department of Physics, University of Utah, Salt Lake City, Utah 84112
}

(Received 25 February 1999)

\begin{abstract}
We introduce a version of the $\mathrm{cw}$ photomodulation technique, measured far from the steady state, for obtaining the quantum efficiency, $\eta$, of long-lived photoexcitations in $\pi$-conjugated polymers. We apply this technique to films of a ladder-type poly(para-phenylene) [mLPPP] for studying the photogeneration action spectra, $\eta(E)$, and recombination kinetics of photogenerated neutral and charged excitations such as singlet and triplet excitons and charged polarons. Whereas the $\eta(E)$ spectrum for singlet excitons shows a step function increase at a photon energy, $E$, close to the optical gap $(\simeq 2.6 \mathrm{eV})$, both triplet and polaron $\eta(E)$ spectra show, in addition, a monotonous rise at higher $E$. The rise for triplets is explained by singlet exciton fission into triplet pairs, and from a model fit we get the triplet exciton energy $(\simeq 1.6 \mathrm{eV})$. For polarons this rise is modeled by an electron intersegment tunneling process. The electroabsorption spectrum is also measured and analyzed in terms of Stark shift of the lowest lying exciton, $1 B_{u}$, and enhanced oscillator strength of the important $m A_{g}$ exciton. A consistent picture for the lowest excited state energy levels and optical transitions in the neutral (singlet and triplet) and charged manifolds is presented. From both the exciton binding energy of $\simeq 0.6 \mathrm{eV}$ and the singlet-triplet energy splitting of $\simeq 1 \mathrm{eV}$, we conclude that the $e$-e interaction in mLPPP is relatively strong. Our results are in good agreement with recent $a b$ initio band structure calculations for several $\pi$-conjugated polymers. [S0163-1829(99)13531-8]
\end{abstract}

\section{INTRODUCTION}

The photogeneration dynamics of singlet excitons and secondary photoexcitations, such as triplets and polarons, in $\pi$-conjugated polymers have usually been measured by picosecond (ps) transient spectroscopic techniques rather than cw spectroscopies, since their photogeneration processes usually occur in the subnanosecond time domain. ${ }^{1}$ On the other hand, the excited states energy levels are often measured in these polymers by $\mathrm{cw}$ optical techniques, including electroabsorption ${ }^{2,3}$ and resonant Raman scattering, ${ }^{4-6}$ and by two and three photon nonlinear optical spectroscopies. ${ }^{7,8}$ In this paper we introduce a version of $\mathrm{cw}$ photomodulation action spectroscopy, which is capable of measuring the photogeneration dynamics of secondary photoexcitations without the need of ps transient optical techniques. This technique uses the excitation dependence (action spectrum) of various photoinduced absorption (PA) bands in the photomodulation spectrum, measured under conditions far from the steady state.

We have used the PA action spectrum technique to obtain the photogeneration quantum efficiency, $\eta$, and its dependencies on temperature, $\theta$, and excitation photon energy, $E$, for singlet and triplet excitons and polarons, respectively, in films of methyl substituted ladder-type poly(para-phenylene) [mLPPP] (Fig. 1, inset). mLPPP is an attractive $\pi$-conjugated polymer for blue-light emitting diodes ${ }^{9,10}$ (see Fig. 1) and photopumped lasers ${ }^{11,12}$ due to its high photoluminescence quantum yield; this is caused in part, by the intrachain order in the film induced by the planarization of neighboring phenyl rings ${ }^{13}$ (Fig. 1 inset). We found that whereas the action spectrum for the photoluminescence quantum efficiency comprises a step-function response at $E$ close to the optical gap, $E_{\mathrm{op}} \simeq 2.6 \mathrm{eV}$, the $\eta(E)$ spectrum for both triplet exciton and polaron PA bands also contains a monotonous rise at $E>E_{\mathrm{op}}$. For triplet excitons this rise is interpreted as due to singlet exciton fission into triplet pairs, and from a model fit to the experimental data we get the triplet energy, $E_{T} \simeq 1.6 \mathrm{eV}$. The rise in the $\eta(E)$ spectrum of polarons at high energies is explained by a hot exciton dissociation process via electron intersegment tunneling. We also measured the electroabsorption spectrum and analyze it in terms of electric field induced changes of odd and even parity excitons. From these measurements a consistent picture of the most important excited energy levels and optical transitions is constructed. 


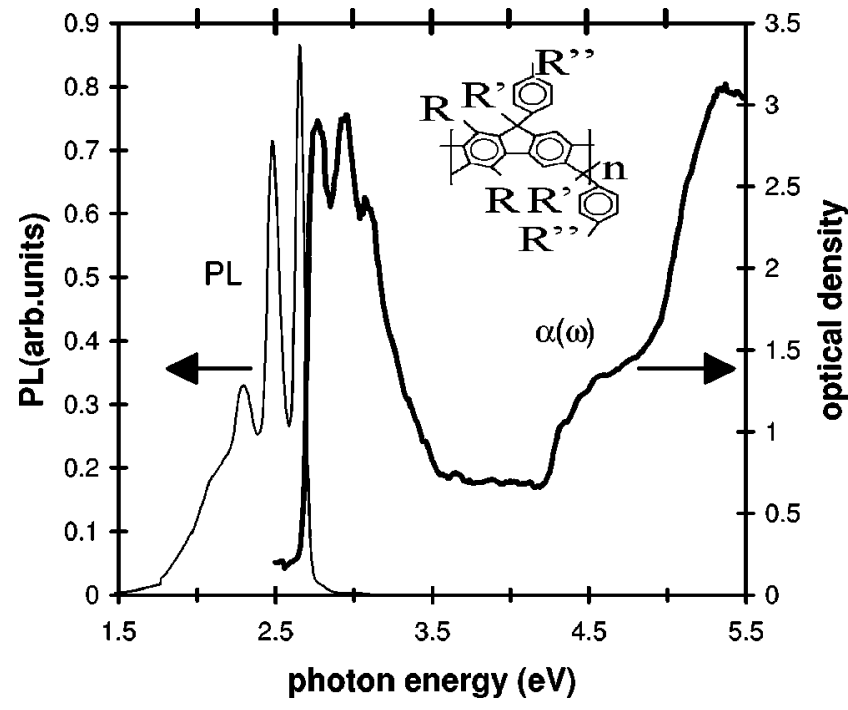

FIG. 1. Photoluminescence (PL, solid) and absorption $[\alpha(\omega)$, bold] spectra of mLPPP. Inset shows the mLPPP repeat unit.

\section{EXPERIMENT}

Our method is based on the cw photomodulation (PM) technique. For the excitation beam we used either an $\mathrm{Ar}^{+}$ laser at several discrete photon energies, $E$, or a monochromatized Xenon lamp to continuously vary $E$, between 2 and $4.5 \mathrm{eV}$. The excitation beam was modulated with a chopper at frequencies, $f$, between 10 and $4 \mathrm{kHz}$. A combination of various incandescent lamps (tungsten halogen and glowbar), diffraction gratings, optical filters, and solid state detectors (silicon, germanium, indium antimonide, and mercury cadmium teluride) was used to span the probe photon energy, $\hbar \omega$, between 0.1 and $3 \mathrm{eV}$. The PM spectrum vs $\hbar \omega$ was obtained by dividing the pump beam induced changes in the probe beam transmission, $\Delta T(\omega)$, by the probe beam transmission, $T(\omega)$, where $\Delta T$ was measured by a phase sensitive technique; in this case the PA, or $\Delta \alpha\left(=-d^{-1} \Delta T / T\right.$, where $d$ is the film thickness) does not depend on the system energy response. $\Delta T$ was measured in our studies as a function of pump excitation intensity, $I$, modulation frequency, $f$, and temperature, $\theta$. To obtain the quantum efficiency per absorbed photon we multiplied $I$ by the factor $g(E)=1$ / $(E d)(1-R)[1-\exp (-\alpha d)]$, where $R(E)$ and $\alpha(E)$ are the film reflectivity and absorption coefficient, respectively.

The $\mathrm{PA}$, or $\Delta \alpha$, is proportional to the photoexcitation density, $N$, via the relation $\Delta \alpha=N \sigma$, where $\sigma$ is the excitation optical cross section. Since $\Delta \alpha \sim \Delta T$, it follows that the changes in the probe transmission measured in phase, $\Delta T_{\text {in }}$, and quadrature, $\Delta T_{Q}$, to the laser beam modulation may be directly related to $N$ dynamics, where $\Delta T_{\text {in }} \sim N_{\text {in }}$ and $\Delta T_{Q}$ $\sim N_{Q}$. The two $N$ components, $N_{\text {in }}$ and $N_{Q}$, may be obtained, in principle, from the modulated excitation intensity $I(t)$, which is a periodic square wave in time with an illuminating pulse duration $t_{0}=1 / 2 f$, where $f$ is the laser modulation frequency, using either the monomolecular (MR) or bimolecular $(\mathrm{BR})$ rate equation:

$$
\frac{d N}{d t}=\eta g I-\beta N \quad(\mathrm{MR})
$$

TABLE I. Photoexcitation density $N$ in the photomodulation measurements for monomolecular (MR) and bimolecular recombination (BR) kinetics in the limits of steady state and far from the steady state. $N_{\text {in }}, N_{Q}$ are the in-phase and quadrature photoexcitation densities, respectively. $g I$ gives the density of absorbed photons, $\eta$ is the photogeneration quantum efficiency, $\tau$ is the MR photoexcitation decay time, and $\gamma$ is the bimolecular recombination constant.

\begin{tabular}{lcc}
\hline \hline Recombination kinetics & Steady state & $\begin{array}{c}\text { Far from the } \\
\text { steady state }\end{array}$ \\
\hline Monomolecular & $N_{\text {in }}=\eta g I \tau / 2$ & $N_{\text {in }}=O\left[1 /\left(\tau f^{2}\right)\right]$ \\
Bimolecular & $N_{Q}=O\left(\tau^{2} f\right)$ & $N_{Q}=\eta g I / 4 \pi f$ \\
& $N_{\text {in }}=\sqrt{\eta g I / 2 \gamma}$ & $N_{\text {in }}=O\left[1 /\left(\tau f^{2}\right)\right]$ \\
& $N_{Q}=O\left(\tau^{2} f\right)$ & $N_{Q}=\eta g I / 4 \pi f$ \\
\hline \hline
\end{tabular}

$$
\frac{d N}{d t}=\eta g I-\gamma N^{2} \quad(\mathrm{BR})
$$

In Eqs. (1) and (2), $\beta$ and $\gamma$ are the MR and BR constants, which are related to the photoexcitation decay time $\tau$ by $\tau$ $=1 / \beta$ and $\tau=1 /(\gamma N)$, respectively. We calculated (see the appendix and Table I) complete analytic expressions for the two $N$ components for the MR kinetics, and analytic expressions for the BR kinetics in the limiting cases of steady state $(f \tau \ll 1)$ and far from the steady state $(f \tau \gg 1)$. Moreover, the exact numerical results for the BR kinetics were also obtained. As a particularly important result of these calculations we found that far from the steady state, namely $f \tau$ $\gg 1$,

$$
N_{Q}=\frac{\operatorname{Ig} \eta}{4 \pi f},
$$

independent of $\tau$ and the recombination kinetics. The decay time $\tau$ depends on the type of recombination kinetics and on various external parameters such as temperature and most importantly in the case of BR kinetics, on the excitation density. Since the excitation density strongly varies in the experiment (it is determined by the lamp intensity, the grating efficiency, the sample reflectivity and absorption coefficient dependencies on $E$ ) then the absence of $\tau$ in the above expression for $N_{Q}$ is essential for the direct determination of $\eta$ from cw measurements. Thus, when measuring $N_{Q}$ far from the steady state, studies of the photoexcitation generation process via the $E$ and $\theta$ dependencies of $\eta$ can be readily achieved with $\mathrm{cw}$ techniques. We note that in previous studies, ${ }^{14-16} N_{\text {in }}(f)$ and $N_{Q}(f)$ were analyzed for both MR and BR kinetics; however, that $N_{Q}$ for $f \tau \gg 1$ does not depend on $\tau$ was not realized and thus basically ignored. Therefore, it has been generally believed that $\mathrm{cw}$ techniques cannot be used to directly study $\eta$ for various photoexcitations since, in contrast to $N_{Q}$ (see the appendix), $N_{\text {in }}$ depends on $\tau$, which, in turn, depends on $E$ and $\theta$. This is, however, not the case if $N_{Q}$ is measured far from the steady state [Eq. (3)].

We summarize the results of our calculations for $N_{\text {in }}$ and $N_{Q}$ in Table I. Also, the characteristic properties of $N_{\text {in }}(f)$ and $N_{Q}(f)$ are demonstrated in Fig. 2, where the calculated $N_{Q}(f)$ and $N_{\text {in }}(f)$ normalized by $G=g \eta I$, are plotted for both MR and BR, respectively, with two different recombi- 


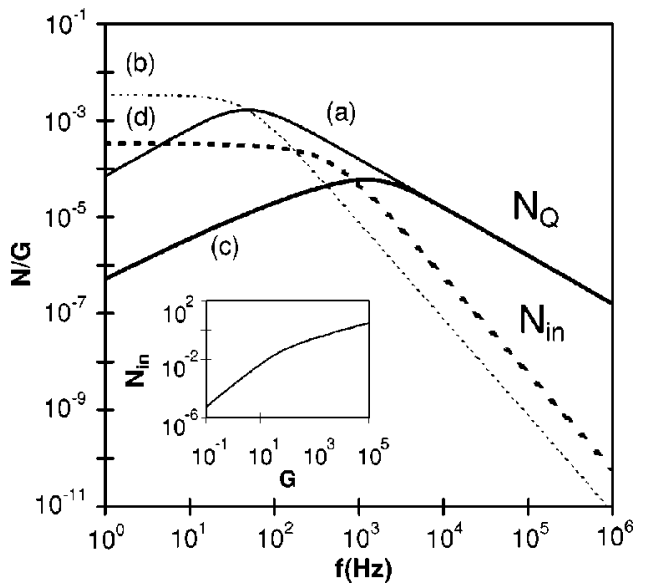

FIG. 2. The two photoexcitation density components $N_{Q}$ and $N_{\text {in }}$ normalized by the absorbed photon density $G$, calculated vs modulation frequency $f$. Curves $(a)$ and $(b)$ are for MR, and curves $(c)$ and $(d)$ are for BR; solid (dotted) lines are for $N_{Q}\left(N_{\text {in }}\right)$. The recombination constants are $\beta=300 \mathrm{~Hz}$ and $\gamma N=1 \mathrm{kHz}$, for the MR and BR kinetics, respectively. The inset shows $N_{\text {in }}$ vs $G$ calculated for BR kinetics with $\gamma N=1 \mathrm{kHz}$ and $f=100 \mathrm{~Hz}$.

nation rates. Firstly, it is seen in Fig. 2 that $N_{\text {in }}=N_{Q}$ at $f_{0}$, such that $f_{0} \tau \simeq 1$, for both recombination processes. Secondly, at $f \gg f_{0}, N_{Q} \sim 1 / f$, whereas $N_{\mathrm{in}} \sim 1 / f^{2}$, and therefore $N_{Q}$ dominates $|\Delta T|$ at high $f$. Importantly, $N_{Q}(f)$ approaches the asymptotic behavior, $1 / f$, at large $f$, independent of the recombination process and $\tau$. Thirdly, at $f>f_{0}, N_{\text {in }}(f)$ scales with $1 / \tau$, explaining the reason why $N_{\text {in }}(\mathrm{BR})$ $>N_{\text {in }}(\mathrm{MR})$ at high $f$, whereas the opposite is true at low $f$.

To identify the recombination kinetics of various excitations in the PM spectrum, we also calculated $N_{\text {in }}(G)$ at a fixed $f$ (Fig. 2, inset). We found that $N_{\text {in }}$ increases linearly with $G$ for the MR kinetics. However, for the BR kinetics we calculated $N_{\text {in }} \sim G^{3 / 2}$ at low $G$ (where $f \tau \gg 1$ ) changing at high $G$ (where $f \tau \ll 1$ ) to $\sqrt{G}$ dependence. We therefore note that the $N_{\text {in }}$ vs $G$ plot may serve to identify the photoexcitation recombination kinetics using $\mathrm{cw}$ studies, whereas $N_{Q}$ measured far from the steady state may be used to directly obtain $\eta$ for the various photoexcitations in the PM spectrum.

The spin state of these excitations may be obtained by the PA detected magnetic resonance (PADMR) technique. ${ }^{17,18} \mathrm{In}$ PADMR we measure the changes in PA induced by a modulated $\mu$-wave field (in our experiment, $3 \mathrm{GHz}$ ) in resonance with the Zeeman split spin-1/2 sublevels in magnetic field $H .^{17,18}$ The $\mu$-wave resonant absorption leads to small changes, $\delta T$, in $T$. This $\delta T$ is proportional to $\delta N$ induced by the $\mu$ waves, caused by changes in spin-dependent recombination rates. Two types of PADMR spectra are possible: ${ }^{18}$ The $H$-PADMR spectrum, in which $\delta T$ is measured at a fixed probe wavelength $\lambda$ as the magnetic field $H$ is scanned, and the $\lambda$-PADMR spectrum, in which $\delta T$ is measured at a constant $H$, in resonance, while $\lambda$ is scanned.

The measurements in this paper were done on a mLPPP film that was drop-cast from a toluene solution on a sapphire substrate and its optical density, (OD), at the laser wavelength was $\approx 1$ (Fig. 1).

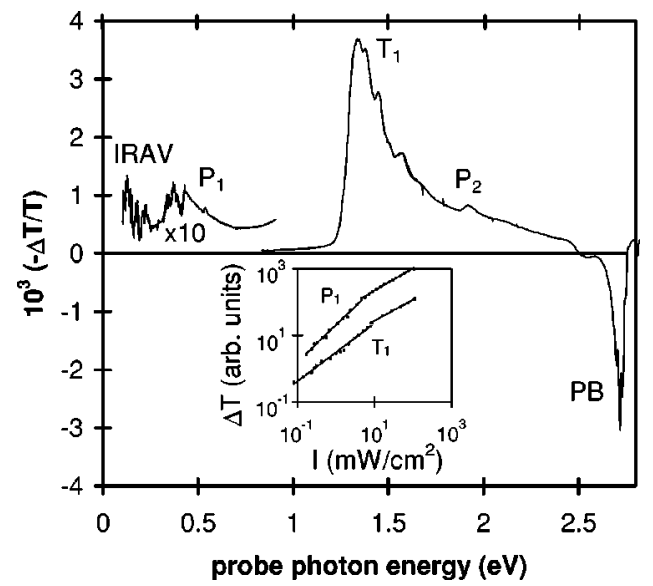

FIG. 3. The in-phase photomodulation spectrum of a mLPPP film at $80 \mathrm{~K}$, excited at $3.5 \mathrm{eV}$ and modulated at $100 \mathrm{~Hz}$. The triplet $\left(T_{1}\right)$, polaron $\left(P_{1}\right.$ and $\left.P_{2}\right)$, IR active vibrations (IRAV) and photobleaching $(\mathrm{PB})$ bands are assigned. The inset shows the dependencies of $T_{1}$ and $P_{1}$ PA bands on the excitation intensity $I$, where the lines are power laws with exponents close to 1 and 0.5 , respectively.

\section{RESULTS AND DISCUSSION}

In Fig. 1 we show the polymer repeat unit, the optical absorption, $\alpha(\omega)$ and photoluminescence (PL) spectra of the mLPPP film used here. At low photon energies, $\alpha(\omega)$ consists of a three peak structure at $2.75,2.93$, and $3.11 \mathrm{eV}$, respectively, that we interpret as an optical transition into the lowest odd parity exciton $\left(1 B_{u}\right)$ and its two phonon replica $\simeq 180 \mathrm{meV}$ apart. The PL spectrum is much sharper than $\alpha(\omega)$, but otherwise also contains a pronounced three peak feature, which is Stokes shifted from that in $\alpha(\omega)$ by about $0.1 \mathrm{eV}$. Using an integrating sphere, we measured the absolute PL quantum efficiency in mLPPP to be about $30 \%$. At high photon energies, $\alpha(\omega)$ also contains absorption bands at 4.5 and $5.3 \mathrm{eV}$, respectively, that are interpreted as due to transitions into higher, but more localized excitons. ${ }^{19}$

\section{A. PM spectrum}

In Fig. 3 we show the PM spectrum of a drop-cast mLPPP film at $\theta=80 \mathrm{~K}$ excited at $E=3.5 \mathrm{eV}$. The PM spectrum is dominated by the PA band $T_{1}$ at $1.3 \mathrm{eV}$ and also by the two correlated PA bands: $P_{1}$ and $P_{2}$ at 0.4 and $1.9 \mathrm{eV}$, respectively. A series of photoinduced infrared active vibrations (IRAV's) that are correlated by their $f$ and $\theta$ dependencies with the PA bands $P_{1}$ and $P_{2}$, but not with $T_{1}$, are also seen at $\hbar \omega<0.25 \mathrm{eV}^{20,21}$ The photobleaching (PB) feature in Fig. 3 marks the mLPPP optical gap, $E_{\mathrm{op}} \simeq 2.6 \mathrm{eV}, 22$ which can also be deduced from the PL band onset at high $\hbar \omega$ (Fig. 1). The photoinduced IRAV's indicate that charge carriers are photogenerated in the polymer chains. Their correlation with $P_{1}$ and $P_{2}$, but not with $T_{1}$ shows, therefore, that the former bands are due to long-lived charged excitations, whereas the latter band is caused by long-lived neutral excitations.

\section{B. PADMR spectroscopy}

The $H$-PADMR spectrum of mLPPP at $\hbar \omega=1.9 \mathrm{eV}$ is shown in Fig. 4(a), inset. We observe a negative resonance at 


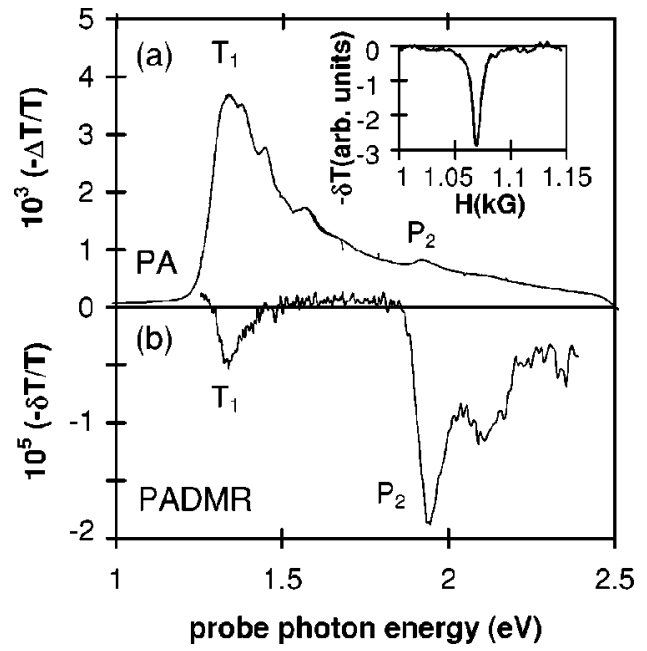

FIG. 4. PA spectrum (a) compared to $\lambda$-PADMR spectrum (b) of spin-1/2 excitations, measured at $H=1065 \mathrm{G}(g=2)$ and $10 \mathrm{~K}$. The inset shows the H-PADMR spectrum at $1.9 \mathrm{eV}$.

$1065 \mathrm{G}$ due to enhanced recombination of spin-1/2 photoexcitations. The $\lambda$-PADMR spectrum measured at $H$ $=1065 \mathrm{G}$ is shown in Fig. 4(b). It contains a sharp band at $1.9 \mathrm{eV}$, which coincides in energy with the $P_{2}$ band [Fig. 4(a)], followed by a phonon replica at $2.1 \mathrm{eV}$. We note the remarkable ability of PADMR to elucidate small PA bands such as $P_{2}$, which are covered by much stronger bands, such as $T_{1}$ in the PM spectrum [compare Figs. 4(a) and 4(b)]. The $\lambda$-PADMR spectrum also shows that $T_{1}$ is much less correlated with spin-1/2 excitations and therefore does not originate from long-lived polarons. We also note that $P_{1}$ and $P_{2}$ bands, in fact coincide in energy with the two doping induced absorption bands in mLPPP caused by polarons, ${ }^{20}$ and that $T_{1}$ is close in energy to the long-lived excitations in isolated PPP oligomers assigned to triplets. ${ }^{21}$ Based on these facts and the spectroscopies described above, we conclude that $P_{1}$ and $P_{2}$ PA bands are due to photogenerated polarons, whereas $T_{1}$ is caused by photoexcited triplet excitons. In the following we use these assignments to study the long-lived photoexcitation generation and recombination processes in $\mathrm{mLPPP}$.

The fact that $T_{1}$ also shows a negative spin- $1 / 2$ resonance, although weak, is interesting by itself and shows that there exists a correlation between polarons and triplet photoexcitations in mLPPP. This may be explained by a model of triplet photogeneration, in which two spin-parallel polarons undergo fusion to become a triplet exciton. ${ }^{23}$ This explains why both resonances, at $P_{2}$ and $T_{1}$, respectively, have the same sign: reducing the density of polarons by magnetic resonance absorption that enhances their recombination rate also results in a reduced density of triplets. However, below we will show that polaron fusion is only a minor channel of triplet photogeneration in mLPPP. One could also try to argue that the spin 1/2 PADMR resonance of the triplet PA band is due to the photobleaching of the ground state, which happens because part of the ground state's oscillator strength is stored in the polaron photoexcitations. However, in this scenario, resonance enhanced recombination of the polarons would reduce photobleaching and thus an increase in triplet

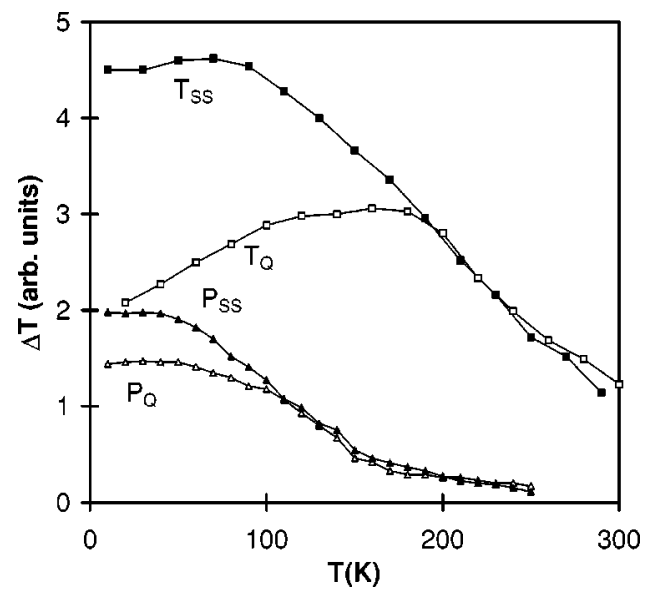

FIG. 5. Temperature dependencies of $T_{1}$ and $P_{1}$ PA bands, both at steady state conditions (measured in-phase, $15 \mathrm{~Hz}$ chopping frequency and $1 \mathrm{~W} / \mathrm{cm}^{2}$ pump intensity), denoted $T_{\mathrm{SS}}$ and $P_{\mathrm{SS}}$, respectively, and far from steady state conditions (measured at quadrature, $4 \mathrm{kHz}, 0.5 \mathrm{~W} / \mathrm{cm}^{2}$ pump intensity), denoted $T_{Q}$ and $P_{Q}$, respectively.

density is expected: The $T_{1}$ would then show a positive resonance, contrary to that observed in the experiment [Fig. 4(b)].

\section{Recombination kinetics}

The inset in Fig. 3 shows the dependence of the in-phase PA for the $T_{1}$ and $P_{1}$ PA bands on the pump laser intensity, measured at $100 \mathrm{~Hz}$. Both bands show a change from a linear dependence at low $I$ to a nearly $\sqrt{I}$. Actually, at high $I$ we measured $\Delta T \sim I^{m}$, where the exponent $m=0.63$ and 0.67 for triplets and polarons, respectively. We calculated that such a change in the exponent $m$ only occurs for the BR kinetics case [Eq. (2)] (see the appendix). We therefore conclude BR kinetics for both polaron and triplet photoexcitations. BR kinetics is obvious for polaron recombination, $P^{+}+P^{-}$ $\rightarrow$ ground state. However, it is not trivial for the photogenerated triplet excitons; our results show, therefore, that triplet-triplet annihilation, where $d T_{1} / d t \sim \gamma T_{1}^{2}$ is dominant in mLPPP, in contrast to triplet exciton kinetics in other polymers. ${ }^{18}$ We note that the neat, planarized mLPPP backbone structure ${ }^{13}$ may increase triplet diffussion in these films, thus promoting BR kinetics. The $T_{1}$ and $P_{1}$ intensity dependencies allow us to rule out polaron fusion as a major channel of triplet photogeneration. Polaron fusion, described above, is a bimolecular generation process (not to be confused with BR), from which it follows that the triplet density depends quadratically on the polaron density; consequently there should be a quadratic relation between their respective PA bands, which is not observed in the experiment.

Figure 5 shows the temperature dependencies of the PA, $\Delta T(\theta)$ of triplets and polarons for both the steady state (SS), $T_{\mathrm{SS}}$, and $P_{\mathrm{SS}}$, respectively, where the PA is measured inphase at $15 \mathrm{~Hz}$ and $I=100 \mathrm{~mW}$, and also far from the steady state, $T_{Q}$ and $P_{Q}$, respectively, where the PA is measured in quadrature at $4 \mathrm{kHz}$ and $I=50 \mathrm{~mW}$. For triplet $\Delta T$ it is seen that for $\theta<200 \mathrm{~K}$ the temperature dependencies of $T_{\mathrm{SS}}$ and $T_{Q}$ are different from each other. However, the two $\Delta T$ have similar $\theta$ dependencies at $\theta>200 \mathrm{~K}$, where steady 


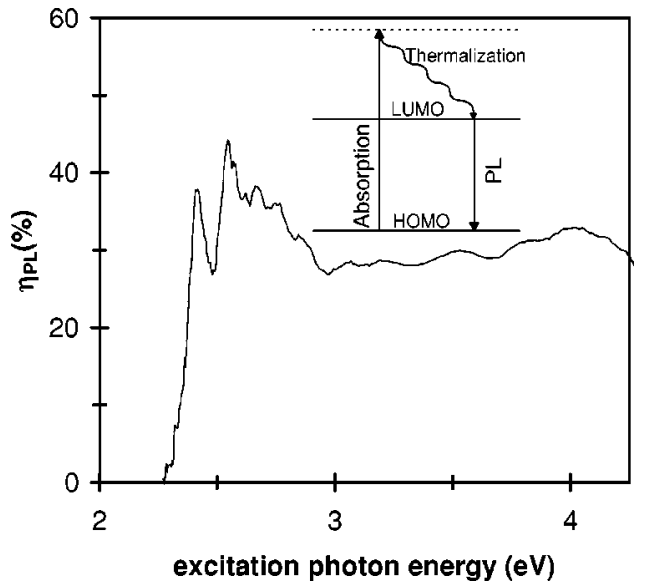

FIG. 6. PL quantum efficiency, $\eta_{\mathrm{PL}}$ vs excitation photon energy. The inset shows that the cw PL is obtained following hot exciton thermalization.

state conditions $(f \tau \leqslant 1)$ are attained. The reason is that at $\theta>200 \mathrm{~K}, \Delta T_{\text {in }}(\theta)$ and $\Delta T_{Q}(\theta)$ are both governed by the temperature dependence of the decay time $\tau(\theta)$ (Table I). Moreover, we note that $T_{Q}$ increases with $\theta$ for $\theta<200 \mathrm{~K}$. Since far from the steady state, $T_{Q}$ is proportional to the photogeneration quantum efficiency $\eta$ [Eq. (3)], then the increase of $T_{Q}$ is caused by an increase of $\eta$ with $\theta$; an unusual $\eta$ property that we can uniquely pick up using our PM technique. For the polarons, both $P_{Q}(\theta)$ and $P_{\mathrm{SS}}(\theta)$ fall on top of each other already for $\theta>100 \mathrm{~K}$, demonstrating that the polaron density is mostly determined by $\tau(\theta)$, whereas the temperature dependence of the generation process itself is much weaker. Indeed, it has been shown ${ }^{24}$ that the temperature dependence of the PA due to charge excitations in mLPPP can be modeled using only the directly, experimentally determined activation energies of traps in mLPPP. Taken together, these results show that the photogeneration quantum efficiency of polarons is temperature independent, in agreement with subnanosecond transient photoconductivity measurement in many other $\pi$-conjugated polymers. ${ }^{25-28}$

\section{Action spectroscopy}

We used the photogeneration action spectrum technique to measure $\eta(E)$ for the PL band and $T_{1}$ and $P_{1}$ PA bands in mLPPP.

\section{PL action spectrum}

By measuring the $\mathrm{PL} /(\mathrm{Ig})$ dependence on the excitation energy, $E$, we obtained the PL quantum efficiency, $\eta_{\mathrm{PL}}(E)$ as shown in Fig. 6. We normalized $\eta_{\mathrm{PL}}(E)$ by the absolute value of $\eta_{\mathrm{PL}}$ measured using an integrating sphere, and found $\eta_{\mathrm{PL}} \approx 30 \%$ at $E=3.5 \mathrm{eV}$. It is seen in Fig. 6 that $\eta_{\mathrm{PL}}(E)$ abruptly increases at $E \approx 2.4 \mathrm{eV}$, followed by a constant value at higher $E$. This step-function behavior is similar to that measured in the best poly(phenylene-vinylene) (PPV) films ${ }^{29}$ and shows that singlet excitons are the primary excitations in mLPPP, as can be also inferred from the large exciton binding energy $(\approx 0.5 \mathrm{eV})$ obtained for this polymer. ${ }^{22} \mathrm{PL}$ mostly occurs following the thermalization of the original hot exciton down to the lowest lying singlet exciton (Fig. 6, inset): Hot excitons thermalize on a times-

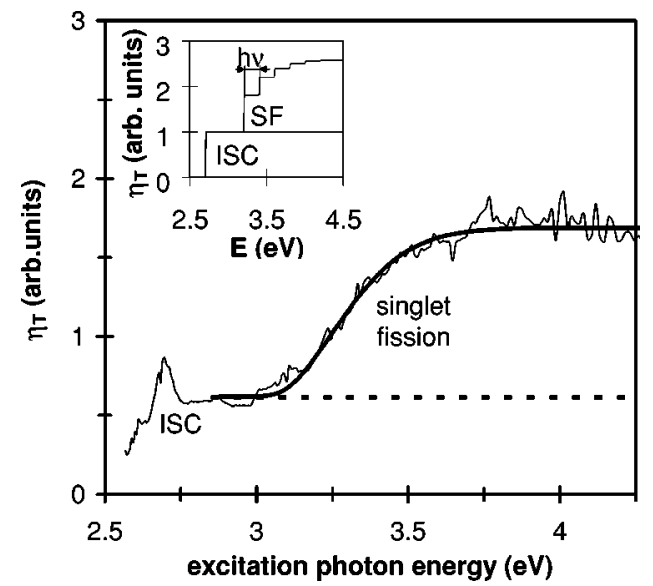

FIG. 7. Photogeneration quantum efficiency spectrum, $\eta_{T}(E)$, of the triplet excitons in mLPPP. The bold line through the data points is a theoretical fit using a model of singlet fission (SF). ISC is the process of triplet photogeneration via intersystem crossing. The dotted line illustrates the separation of $\eta_{T}(E)$ into the two processes. The inset shows an idealized $\eta_{T}(E)$ spectrum with no inhomogeneous broadening (see text), where $h \nu$ is a vibrational quantum.

cale of the order of a ps, whereas the PL emission from thermalized excitons typically occurs on the order of $100 \mathrm{ps}$ following the excitation. ${ }^{30}$ Thus, the singlet exciton recombines radiatively with a given quantum efficiency independent of the original excitation energy. Consequently, the PL action spectrum reflects the photogeneration quantum efficiency spectrum for singlet excitons, which is close to 1 in mLPPP, independent of $E$.

\section{Triplet action spectrum}

At longer times, however, triplet excitons are formed in mLPPP, as may be concluded from the existence of the $T_{1}$ PA band in Fig. 3. By measuring the $\Delta T_{Q} /(\mathrm{Ig})$ dependence on $E$ for the $T_{1}$ band at low $I$ (where $f \tau \gg 1$ ), we obtained the triplet quantum efficiency, $\eta_{T}(E)$ as shown in Fig. 7. Again, $\eta_{T}(E)$ has a step function response at $E \approx E_{\text {op }}$ similar to $\eta_{\mathrm{PL}}(E)$; however, $\eta_{T}(E)$ increases at higher $E$, reaching a plateau at $E \approx 3.7 \mathrm{eV}$. It is thus apparent that triplet generation occurs via two main processes. The first process is associated with the generation of thermalized singlet excitons and therefore has a similar $E$ dependence as that of $\eta_{\mathrm{PL}}(E)$ in Fig. 6. We identify this process as due to intersystem crossing, ISC, from the singlet to the triplet manifold, which occurs following exciton thermalization at times of order 1 ns, ${ }^{30,31}$ and hence its flat response with $E$ for $E \geqslant E_{\text {op }}$. The other process, with an onset at $E \simeq 3.2 \mathrm{eV}$, is therefore due to hot excitons and must thus occur at least on the timescale of the hot exciton thermalization. Both upper excited state transfer $^{32}$ (UEST) and singlet exciton fisson ${ }^{33}$ (SF) mechanisms for triplet generation have onsets above the optical gap. UEST has its maximum efficiency at $E$ close to higher energy triplet states. Then the beginning of the plateau in Fig. 7 should mark the energy of the second lowest triplet exciton state. This energy $(\approx 3.7 \mathrm{eV})$, however, is higher than that of the mAg singlet exciton, which was measured to be at $\simeq 3.3 \mathrm{eV}^{22}$ (see also Sec. III E) and thus UEST cannot explain our data. We therefore identify the second triplet 
photogeneration process as $\mathrm{SF}(E x \rightarrow \mathrm{T} \uparrow+\mathrm{T} \downarrow)$, which is operative at $E \geqslant 2 E_{T}$, where $E_{T}$ is the triplet energy. We thus obtain from the higher $\eta_{T}(E)$ onset at $3.2 \mathrm{eV}$ a value $E_{T}$ $\simeq 1.6 \mathrm{eV}$.

The theoretical prediction for the singlet fission quantum efficiency spectrum is a step function response with the step at $2 E_{T} \cdot{ }^{33}$ But the $\eta_{T}(E)$ spectrum does not show such a sharp step function response (Fig. 7). On the contrary, the rise prior to reaching the plateau is spread out in energy over several tenths of $\mathrm{eV}$. We therefore introduce a model (see also Ref. 34) that is able to explain the shape of this broad rise in energy and get the $E_{T}$ value in spite of this broad spectrum. Our model follows two main ideas: (i) Singlet fission, just like any other electronic transition, may be accompanied by emission of strongly coupled vibrations. (ii) $E_{T}$ is not a single energy, but is spread out due to inhomogeneous broadening, which we denote as $E_{T}(x)$, where the variable $\mathrm{x}$ describes an inhomogeneous parameter, such as conjugation length, for example.

Thus, the energy $E$ necessary to produce a triplet exciton is given by

$$
E=E_{T}(x)+p h \nu_{P},
$$

where $p$ is the number of emitted phonons and $\nu_{P}$ is the vibrational frequency. The relative strength of the emission of $p$ phonons during the electronic transition is described by the Huang-Rhys formula: ${ }^{35}$

$$
h(p)=\frac{S^{p}}{p !} .
$$

Figure 7 inset illustrates the model: For a weak inhomogeneity the SF action spectrum would comprise several steps. The $n$th step marks the onset of the photogeneration of a triplet pair accompanied by the emission of $n$ vibrational quanta.

To quantify the inhomogenous broadening we have fitted the optical absorption band of mLPPP with an asymmetric Gaussian distribution. ${ }^{2}$ Let us call the distribution resulting from this fit $D^{\prime}(E)$, where $D(E)$ is the distribution obtained by summing up over the vibronic progressions. $D(E)$ is then given by $^{2}$

$$
D(E)=\sum_{p=0}^{\infty} h(p) D^{\prime}\left(E+p h \nu_{P}\right)
$$

Singlet fission produces pairs of triplet excitons on neighboring chains or neighboring chain segments. Then the energy $E$ to produce a triplet pair and coupled vibrations is given by

$$
E=E_{T}\left(x_{1}\right)+p_{1} h \nu_{P}+E_{T}\left(x_{2}\right)+p_{2} h \nu_{P},
$$

where the distributions $E_{T}\left(x_{1}\right)=E_{T}\left(x_{2}\right), p_{1}\left(p_{2}\right)$ is the number of emitted phonons in connection with the generation of the first (second) triplet exciton. Next, we convoluted $D(E)$ for the two triplet excitons to obtain the distribution $D_{\text {pair }}$ describing the inhomogenous distribution and phonon emission related to the generation of a triplet pair with energy $E_{\text {pair }}$ :

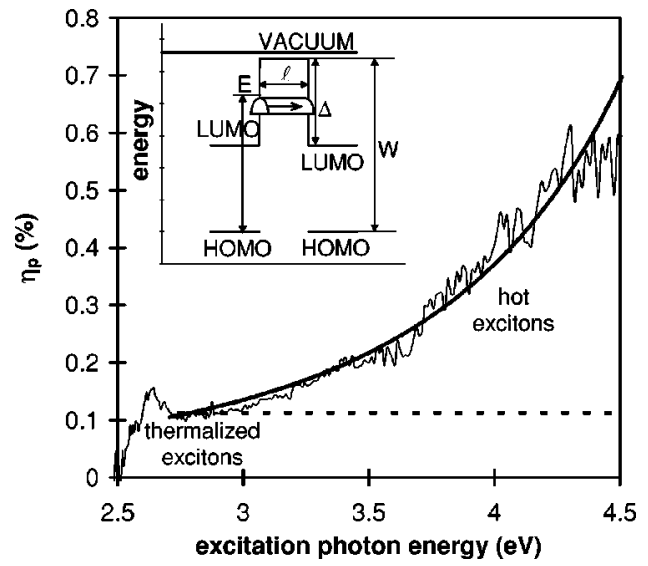

FIG. 8. Photogeneration quantum efficiency spectrum, $\eta_{P}(E)$, of polaron excitations in mLPPP; the two photogeneration processes due to hot and thermalized excitons, respectively, are assigned. The dotted line illustrates the separation of $\eta_{P}(E)$ into the two processes. The line through the data points is a theoretical fit using an intersegment tunneling model, as explained in the inset. HOMO (LUMO) is the highest occupied (lowest unoccupied) molecular orbital, $l(\Delta)$ is the intersegment barrier potential width (height), and $W$ is the energy difference between the HOMO and the vacuum level.

$$
D_{\text {pair }}\left(E_{\text {pair }}\right)=\int_{E_{1}=0}^{E_{\text {pair }}} D\left(E_{1}\right) D\left(E_{\text {pair }}-E_{1}\right) d E_{1} .
$$

We get the triplet photogeneration quantum efficiency, $\eta_{T}$, by convoluting $D_{\text {pair }}$ with a step function having an onset at $E=E_{\text {pair }}$.

$$
\begin{aligned}
\eta_{T}(E) & =\int_{E_{\text {pair }}=0}^{\infty} D_{\text {pair }}\left(E_{\text {pair }}\right) H\left(E-E_{\text {pair }}\right) d E_{\text {pair }}, \\
& =\int_{E_{\text {pair }}=0}^{E} D_{\text {pair }}\left(E_{\text {pair }}\right) d E_{\text {pair }}, \\
& =\int_{E_{\text {pair }}=0}^{E} \int_{E_{1}=0}^{E_{\text {pair }}} D\left(E_{1}\right) D\left(E_{\text {pair }}-E_{1}\right) d E_{1} d E_{\text {pair }},
\end{aligned}
$$

which finally results in

$$
\eta_{T}(E)=\int_{E_{2}=0}^{E} \int_{E_{1}=0}^{E-E_{2}} D\left(E_{1}\right) D\left(E_{2}\right) d E_{1} d E_{2},
$$

where $E_{2}=E_{\text {pair }}-E_{1}$.

Equation (10) contains $E_{T}$ and $S$ as the only two fitting parameters since $D(E)$ is directly extracted from $\alpha(\omega)$. The excellent fit to the experimental $\eta_{T}(E)$ curve shown in Fig. 7 was obtained using Eq. (10) with $E_{T}=1.6 \mathrm{eV}$ and $S=0.15$. From this measurement we note that the singlet-triplet energy difference $\Delta_{\mathrm{ST}}=E_{S}-E_{T}$ is approximately $1 \mathrm{eV}$ for the lowest exciton $\left(1 B_{u}\right)$, and that similar values for $\Delta_{\mathrm{ST}}$ were also measured in PPV, ${ }^{36}$ as well as calculated by recent $a b$ initio band structure calculations. ${ }^{37}$

\section{Polaron action spectrum}

In Fig. 8 we show the polaron photogeneration quantum efficiency, $\eta_{P}(E)$ obtained by measuring $\Delta T_{Q}(E) /(\operatorname{Ig})$ for 
the $P_{1}$ PA band (Fig. 3), and normalizing it by the doping induced absorption cross section, $\sigma$, of polarons. ${ }^{20}$ It is seen that $\eta_{P}(E)$ abruptly increases at $E=2.5 \mathrm{eV}$, similar to $\eta_{\mathrm{PL}}(E)$ and $\eta_{T}(E)$ in Figs. 6 and 7, respectively. At $E$ $>2.85 \mathrm{eV}$, however, $\eta_{P}(E)$ monotonously increases with $E$, where a saturation at high $E$ similar to that found for $\eta_{T}(E)$ in Fig. 7 is not observed. It is again obvious that $\eta_{P}(E)$ is composed from two contributions related to two different polaron photogeneration processes. One process is due to thermalized excitons and is thus independent of $E$, similar to $\eta_{\mathrm{PL}}(E)$ (Fig. 6). We identify this process as exciton dissociation $\left(E X \rightarrow P^{-}+P^{+}\right)$at impurities and defects in the film, which occurs following hot exciton thermalization, similar to the "extrinsic" process observed in cw photoconductivity (PC) ${ }^{38-40}$ The second polaron photogeneration process showing a distinctive $E$ dependence is related to hot excitons and is thus intrinsic in nature. ${ }^{41}$ To explain this temperature independent, intrinsic process we suggest an electron tunneling model (Fig. 8 inset), as follows:

The electron and hole of a thermalized exciton are closely bound by a binding energy of $\approx 0.6 \mathrm{eV}$ (see Sec. III E). This makes their separation into free charges (polarons) very unlikely; soon after its thermalization the exciton will recombine to the ground state. We argue that this immediate recombination can only be prevented by separating the electron and hole to neighboring chain segments during the exciton thermalization. We therefore suggest that at initially high $E$ the electron may tunnel to another chain segment, before its excess energy is completely released. The tunneling probability for the electron, $p(E)$, is given by

$$
p(E)=\nu_{0} \int_{E_{\mathrm{op}}}^{E} e^{-2 l / \hbar \sqrt{2 m_{e}^{*}\left(\Delta+E_{\mathrm{op}}-E^{\prime}\right)}} d E^{\prime},
$$

where $l$ is the tunneling barrier thickness, $m_{e}^{*}$ is the electronic effective mass, and $\Delta$ is the barrier height for tunneling; here $\Delta+E_{\mathrm{op}}=W$, where $W$ is the polymer highest occupied molecular orbital (HOMO)-vacuum energy difference (see Fig. 8, inset); $\nu_{0}$ is the number of tunneling attempts per unit energy as the electron thermalizes from $E$ to $E_{\mathrm{op}}$. The integrand in Eq. (11) is the standard WKB formula for the tunneling probability through a square potential barrier. As polaron photogeneration happens during the thermalization process, the tunneling probability is integrated over the thermalization time, where $\nu_{0}$-assumed to be a constantestablishes the connection between the time and energy scale. This tunneling model is in agreement with the directly determined time evolution (with 100 fs resolution) of the exciton dissociation probability in $\mathrm{mLPPP},{ }^{42}$ which is one order of magnitude higher during hot exciton thermalization as compared to thermalized excitons. The following values for $\nu_{0}, W$, and $l \sqrt{m_{e}^{*}}$ are extracted from the fit obtained to both $\eta_{P}(E)$ spectrum and magnitude shown in Fig. 8: We obtained $\nu_{0}=0.5(\mathrm{eV})^{-1}, W=5.5 \pm 0.5 \mathrm{eV}$, which is in excellent agreement with electron affinity measurements in $\operatorname{mLPPP},{ }^{10} l \sqrt{m_{e}^{*}}=4 \pm 1 \AA \sqrt{m_{e}}$. The limits for the intrachain $m_{e}^{*}$ value are $0.1 m_{e}$ (conventional inorganic semiconductors) and $m_{e}$; for these values of $m_{e}^{*}$ we obtained from $l \sqrt{m_{e}^{*}}$ determined above $l=12$ and $l=4 \AA$, respectively. These values are in agreement with the expected barrier

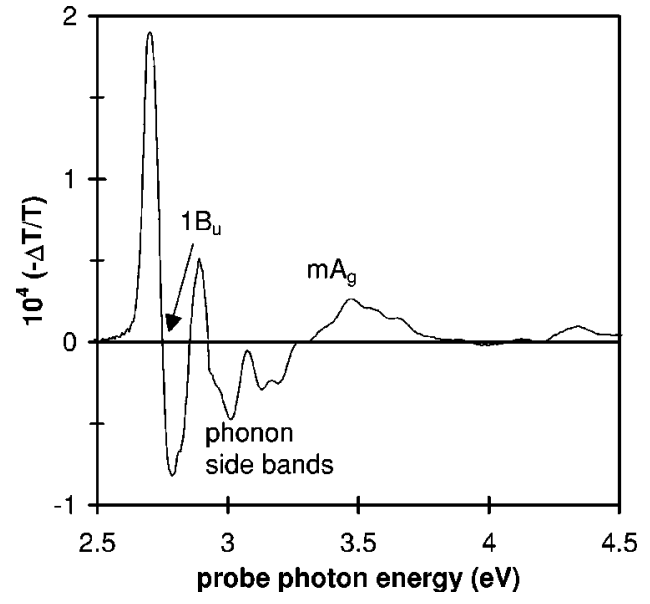

FIG. 9. Electroabsorption spectrum of a mLPPP film at $80 \mathrm{~K}$. The spectral features associated with the Stark shift of the $1 B_{u}$ exciton, its phonon replica, and the $m A_{g}$ exciton are assigned.

thickness separating two conjugation segments. It is known that the maximum energy relaxation rate $R=\Delta E / \Delta t$ of hot excitons is $R=h \nu^{2}{ }^{43}$ where $\nu$ is the most strongly coupled phonon. For mLPPP $h \nu=0.18 \mathrm{eV}(C=C$ stretching mode), determined from the $\alpha(\omega)$ replica in Fig. 1. We can therefore estimate the attempt frequency for tunneling, $\nu_{T}$, from the relation

$$
\nu_{T}=R \nu_{0} .
$$

From $\nu_{0}=0.5 \mathrm{eV}^{-1}$ determined above and Eq. (12), we get $\nu_{T} \approx 0.1 \nu$, which is reasonably close to the maximum allowed $\nu_{T}(=\nu)$.

After thermalization is completed electron tunneling is not possible any more. Indeed, there is an energy onset to electron tunneling, as it must be at least equal to the difference between exciton and polaron pair binding energies. We note that at electric fields, $F$, of order $10^{5} \mathrm{~V} / \mathrm{cm}$, a similar model was used to explain $\eta_{\mathrm{PC}}(E)$ at high $F{ }^{44}$ where the electron and hole are separated by a barrier arising from their Coulomb interaction and $F$.

\section{E. Electroabsorption spectrum}

To complete the studies of neutral excitations in mLPPP we also measured the electroabsorption (EA) spectrum. The measurements were made using a modulated electric field of up to $1.6 \times 10^{5} \mathrm{~V} / \mathrm{cm}$. The mLPPP film was deposited on an electrode structured sapphire substrate consisting of interlocking metallic fingers with $20 \mu \mathrm{m}$ gap. The electric field modulation frequency was $500 \mathrm{~Hz}$, and the changes $\Delta T$ in transmission $T$ due to the applied voltage were measured with a lock-in amplifier at $2 f .^{2}$

The EA spectrum of an mLPPP film at $80 \mathrm{~K}$ is shown in Fig. 9 up to $4.5 \mathrm{eV}$. The spectrum consists of three derivativelike features with peaks at 2.7, 2.88, and $3.06 \mathrm{eV}$, respectively, and a positive spectral feature with an onset at 3.3 $\mathrm{eV}^{.22}$ the highest energy bump at $4.3 \mathrm{eV}$ is due to higher excitons in $\alpha(\omega)$ (Fig. 1). Similar spectral features have been observed before in the EA spectra of many $\pi$-conjugated polymers ${ }^{2}$ and thus we analyze these features according to the standard model using summation over 
(a)

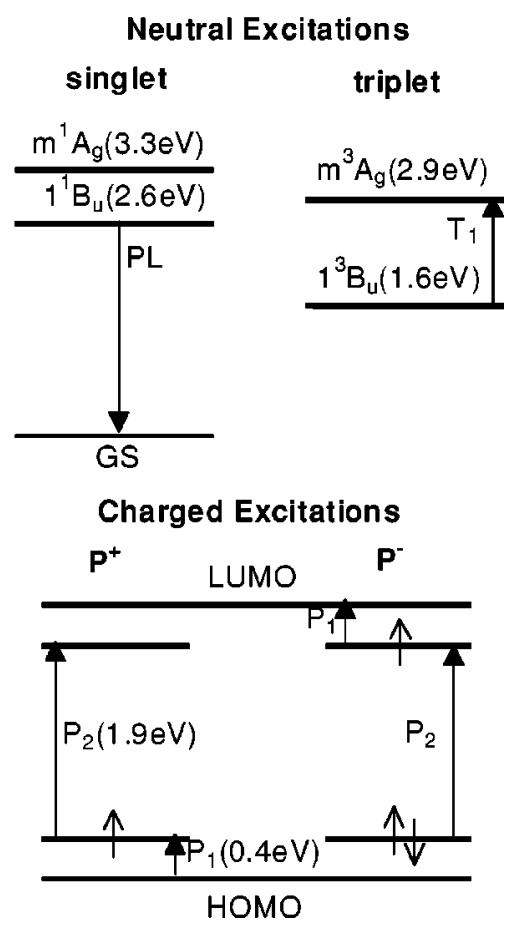

FIG. 10. Schematic representation of the excited states energy levels and optical transitions of the most important excitons with odd $\left(B_{u}\right)$ and even $\left(A_{g}\right)$ parity and polarons in mLPPP. (a) Neutral singlet and triplet manifold. (b) Polaronic levels in the charged manifold for both $P^{+}$and $P^{-}$.

states. ${ }^{2,45}$ We therefore interpret the derivativelike features in Fig. 9 as due to the Stark shift of the $1 B_{u}$ exciton at $\simeq 2.7 \mathrm{eV}$ and its two phonon side bands, which are $180 \mathrm{meV}$ apart. The positive spectral feature at higher energy, on the contrary, is interpreted as due to the $m A_{g}$ exciton at $\simeq 3.3 \mathrm{eV}$. We note that the $1 B_{u}$ energy deduced here is averaged over the conjugation length distribution in the film, whereas $\mathrm{cw}$ PL is mainly due to the longest chains. This may explain the energy difference between the measured optical gap from the PL onset at $2.6 \mathrm{eV}$, and the average $1 B_{u}$ exciton at $2.7 \mathrm{eV}$ extracted from the EA spectrum.

\section{F. Excited states energy levels}

With these assignments in mind we can now complete the picture of the main excited states energy levels and optical transitions in mLPPP, deduced in this work. This is shown in the schematic representation of Figs. 10(a) and 10(b) for the neutral and charged manifolds, respectively.

In the neutral manifold [Fig. 10(a)] the $1{ }^{1} B_{u}$ at $2.6 \mathrm{eV}$ and the $1^{3} B_{u}$ at $1.6 \mathrm{eV}$ are deduced from the PL band and singlet fission onset, respectively. The $m^{1} A_{g}$ level at $3.3 \mathrm{eV}$ is deduced from the onset of the positive feature in the EA spectrum, whereas the $m^{3} A_{g}$ level at $2.9 \mathrm{eV}$ is deduced from the $T_{1}$ PA band, which theory predicts to be the strongest triplet transition from the $1^{3} B_{u} \cdot{ }^{45}$ From these measurements we note that $\Delta_{\mathrm{ST}}=E_{S}-E_{T}$ is approximately $1 \mathrm{eV}$ for the lowest exciton $\left(1 B_{u}\right)$ and approximately $0.4 \mathrm{eV}$ for the important $m A_{g}$ exciton. That $\Delta_{\mathrm{ST}}$ is smaller for the $m A_{g}$ exciton is consistent with the more extended wave function of this high energy exciton. It also indicates that the continuum band onset, where presumably the singlet and triplet states are degenerate, is located at a still higher energy. This shows that the exciton binding energy, $E_{b}$, defined here as the energy difference between $1{ }^{1} B_{u}$ and the continuum band onset, is at least $0.6 \mathrm{eV}$ in mLPPP, consistent with the relatively strong $e$-e interaction in this polymer. Also, since $\Delta_{\mathrm{ST}}$ is determined by the exchange interaction, then the large $\Delta_{\mathrm{ST}}$ value $(\approx 1 \mathrm{eV})$ for the $1 B_{u}$ exciton obtained from our measurements is in agreement with the strong $e-e$ interaction in mLPPP that we deduced from $E_{b}$. We also note that similar values for $\Delta_{\mathrm{ST}}$ were also measured in $\mathrm{PPV},{ }^{36}$ as well as calculated by recent $a b$ initio band structure calculations. ${ }^{37}$

The HOMO and lowest unoccupied molecular orbital (LUMO) levels in the charged manifold [Fig. 10(b)] do not have to coincide with those of the neutral manifold. In fact the HOMO-LUMO gap, $E_{\mathrm{HL}}$, in PPV was found ${ }^{46}$ to be larger for the charged manifold by about $0.2 \mathrm{eV}$, compared to the optical gap in the neutral manifold. We may estimate $E_{\mathrm{HL}}$ in mLPPP for the charged manifold, if we assume that the polaron levels are symmetrically located in the HL gap, as shown in Fig. 10(b). In this case there exists the following relation:

$$
E_{\mathrm{HL}}=2 P_{1}+P_{2} .
$$

Using $P_{1}=0.4 \mathrm{eV}$ and $P_{2}=1.9 \mathrm{eV}$ from the PA bands in Fig. 3, we calculate from Eq. (13) $E_{H L}=2.7 \mathrm{eV}$. This is close, but higher than $E_{\mathrm{op}}=2.6 \mathrm{eV}$ found in the singlet manifold. That $E_{\mathrm{op}} \approx E_{\mathrm{HL}}$ shows that the polaronic relaxation energy associated with charge injection is small in mLPPP and this is consistent with the relatively high quantum yield found for light emitting diodes (LED's) made from mLPPP thin films.

\section{CONCLUSIONS}

We studied the photoexcitation dynamics as revealed in PM measurements for the cases of monomolecular and bimolecular recombination kinetics, respectively. In particular, we found that the quadrature PA component solely depends on the photogeneration quantum efficiency, if the measurements are conducted far from the steady state. Thus, studies of subnanosecond generation processes of long-lived photoexcitations can be completed using cw PM rather than transient techniques. We presented the PA and PA detected magnetic resonance spectra of mLPPP thin films. The PA spectrum is dominated by a triplet-triplet absorption at 1.3 $\mathrm{eV}$ and by two polaron absorption bands at 0.4 and $1.9 \mathrm{eV}$, respectively. We showed that the band at $1.9 \mathrm{eV}$ has a strong spin-1/2 resonance, whereas for the PA band at $1.3 \mathrm{eV}$ we found a much weaker spin- $1 / 2$ resonance. We proposed a fusion mechanism of two spin-parallel polarons into a triplet to explain the observed triplet spin-1/2 PADMR resonance. The dependencies of the triplet and polaron PA bands on the excitation laser intensity and temperature were measured and analyzed in terms of specific generation and recombination processes. The photogeneration action spectra for the singlet and triplet excitons and polarons were presented. We identified two photogeneration processes for triplets, namely intersystem crossing and singlet fission, and also two photogeneration processes for polarons, namely dissociation at defects and electron intersegment tunneling. From a model fit to the triplet action spectrum we obtained a value for the 
triplet exciton energy, $E_{T}=1.6 \mathrm{eV}$. From fitting the polaron action spectrum we obtained values for the polymer HOMOvacuum energy difference, $W=5.5 \mathrm{eV}$, and for the width of the tunneling barrier, $4 \AA<d<12 \AA$.

From the EA spectrum we found the energy of the two most important excitons in mLPPP, namely $1 B_{u}$ and $m A_{g}$. The energy levels and optical transitions of the most important excitons in the singlet and triplet manifolds and polarons in mLPPP were then deduced and their complete scheme was presented. We conclude that $e-e$ interaction in mLPPP is relatively strong.

\section{ACKNOWLEDGMENTS}

We thank U. Scherf and K. Muellen for the mLPPP powder, and E. Ehrenfreund, I. Gontia, and R. Österbacka for useful discussions. The work at the University of Utah was supported in part by DOE Grant No. FG-03-96 ER 45490, and NSF Grant No. DMR-9732820. This work was also supported by the Austrian FWF SFB, Elektroaktive Stoffe and P.12806 grants, respectively.

\section{APPENDIX}

Assuming that the lock-in amplifier analyzes the first harmonic of $N(t)$, we numerically and analytically solved separately the two rate equations

$$
\frac{d N}{d t}=\eta g I-\beta N(M R) ; \quad \frac{d N}{d t}=\eta g I-\gamma N^{2}(B R),
$$

for $N_{\text {in }}$ and $N_{Q}$ at different $f$ and $I$, spanning the steady state $(f \tau \ll 1)$, and far from it $(f \tau \gg 1)$. The excitation $I(t)$ is a periodic square wave in time with an illuminating pulse duration $t_{0}=1 / 2 f$, where $f$ is the laser modulation frequency, $\tau$ is the photoexcitation decay time, i.e., $\tau=1 / \beta$ and $\tau$ $=1 /(\gamma N)$ for MR and BR kinetics, respectively. An analytic expression for MR kinetics can be obtained by solving the Fourier transform of Eq. (14) for $N(f)$. This gives

$$
N(f)=\frac{\eta g I}{2} \frac{\beta}{(2 \pi f)^{2}+\beta^{2}}-i \frac{\eta g I}{2} \frac{2 \pi f}{(2 \pi f)^{2}+\beta^{2}},
$$

where the real and imaginary parts denote the $N_{\text {in }}$ and $N_{Q}$ components, respectively. We can obtain analytic expressions for BR kinetics in the limiting cases of steady state and far from the steady state. At steady state we set the left-hand side of Eq. (14) to zero and solve for $N(f)$ :

$$
N_{\mathrm{SS}}=\sqrt{\frac{\eta g I}{2 \gamma}} .
$$

Far from steady state (e.g., at high $f$ ) the left-hand side of Eq. (14) becomes important because of the time derivative, however, the recombination term will be negligible, as $N$ decreases with $f$. Under these conditions we can easily solve the Fourier transform of the remaining equation for the component $N_{Q}(f)$ and get

$$
N_{Q}=-\frac{\eta g I}{4 \pi f}
$$

To get the exact, numerical results for the BR kinetics we first solved Eq. (14) analytically in the time domain and calculated the Fourier component integrals numerically. The various terms and their approximations are given in Table I.
${ }^{1}$ Primary Photoexcitations in Conjugated Polymers; Molecular Excitons vs. Semiconductor Band Model, edited by N.S. Sariciftci (World Scientific, Singapore, 1997).

${ }^{2}$ M. Liess, S. Jeglinski, Z.V. Vardeny, M. Ozaki, K. Yoshino, Y. Ding, and T. Barton, Phys. Rev. B 56, 15712 (1997).

${ }^{3}$ M. Cardona, Solid State Physics Supplement 11: Modulation Spectroscopy (Academic Press, New York, 1969).

${ }^{4}$ M. Ozaki, E. Ehrenfreund, R.E. Benner, T.J. Barton, K. Yoshino, and Z.V. Vardeny, Phys. Rev. Lett. 79, 1762 (1997).

${ }^{5}$ Z.V. Vardeny, E. Ehrenfreund, O. Brafman, and B. Horovitz, Phys. Rev. Lett. 51, 2326 (1983).

${ }^{6}$ M. Gussoni, C. Castiglioni, and G. Zerbi, Spectroscopy of Advanced Materials, edited by R.J.H. Clark and R.E. Hester (John Wiley \& Sons, New York, 1991).

${ }^{7}$ W.S. Fann, S. Benson, J.M.J. Madey, S. Etemad, G.L. Baker, and F. Kajzar, Phys. Rev. Lett. 62, 1492 (1989).

${ }^{8}$ B. Lawrence, W.E. Torruellas, M. Cha, M.L. Sundheimer, G.I. Stegeman, J. Meth, S. Etemad, and G. Baker, Phys. Rev. Lett. 73, 597 (1994).

${ }^{9}$ S. Tasch, A. Niko, G. Leising, and U. Scherf, Appl. Phys. Lett. 68, 1090 (1996).

${ }^{10}$ S. Tasch, E.J.W. List, C. Hochfilzer, G. Leising, P. Schlichting, U. Rohr, Y. Geerts, U. Scherf, and K. Müllen, Phys. Rev. B 56, 4479 (1997).

${ }^{11}$ C. Zenz, W. Graupner, S. Tasch, G. Leising, K. Müllen, and U.
Scherf, Appl. Phys. Lett. 71, 2566 (1997).

${ }^{12}$ A. Haugeneder, M. Hilmer, C. Kallinger, M. Perner, W. Spirkl, U. Lemmer, J. Feldmann, and U. Scherf, Appl. Phys. B: Lasers Opt. 66, 389 (1998).

${ }^{13}$ G. Leising, O. Ekstroem, W. Graupner, F. Meghdadi, M. Moser, G. Kranzelbinder, T. Jost, S. Tasch, B. Winkler, L. Athouel, G. Froyer, U. Scherf, K. Muellen, G. Lanzani, M. Nisoli, and S. DeSilvestri, Proc. SPIE 2852, 189 (1996).

${ }^{14}$ C. Botta, S. Luzzati, R. Tubino, D.D.C. Bradley, and R.H. Friend, Phys. Rev. B 48, 14809 (1993).

${ }^{15}$ G. Dellepiane, C. Cuniberti, D. Comoretto, G. Lanzani, G.F. Musso, P. Piaggio, R. Tubino, A. Borghesi, C. Dell'Erba, G. Garbarino, and L. Moramarco, Phys. Rev. B 45, 6802 (1992).

${ }^{16}$ E. Dekel, E. Ehrenfreund, D. Gershoni, P. Boucaud, I. Sagnes, and Y. Campidelli, Phys. Rev. B 56, 15734 (1997).

${ }^{17}$ X. Wei, B.C. Hess, Z.V. Vardeny, and F. Wudl, Phys. Rev. Lett. 68, 666 (1992).

${ }^{18}$ P. Lane, X. Wei, and Z.V. Vardeny, Phys. Rev. B 56, 4626 (1997).

${ }^{19}$ M. Chandross, S. Muzumdar, S. Jeglinski, X. Wei, Z.V. Vardeny, E.W. Kwock, and T.M. Miller, Phys. Rev. B 50, 14702 (1994).

${ }^{20}$ W. Graupner, M. Mauri, J. Stampfl, G. Leising, U. Scherf, and K. Müllen, Solid State Commun. 91, 7 (1994).

${ }^{21}$ W. Graupner, S. Eder, M. Mauri, G. Leising, and U. Scherf, Synth. Met. 69, 419 (1995). 
${ }^{22}$ G. Meinhardt, A. Horvath, G. Weiser, and G. Leising, Synth. Met. 84, 667 (1997).

${ }^{23}$ V. Dyakonov, in Primary Photoexcitations in Conjugated Polymers; Molecular Excitons vs Semiconductor Band Model (Ref. 1), p. 254.

${ }^{24}$ W. Graupner, G. Leditzky, and G. Leising, Phys. Rev. B 54, 7610 (1996).

${ }^{25}$ C.H. Lee, G. Yu, D. Moses, and A.J. Heeger, Phys. Rev. B 49, 2396 (1994).

${ }^{26}$ D. Moses, M. Sinclair, and A.G. Heeger, Phys. Rev. Lett. 58, 2710 (1987).

${ }^{27}$ D. Moses, J. Wang, G. Yu, and A.G. Heeger, Phys. Rev. Lett. 80, 2685 (1998).

${ }^{28}$ D. Moses, H. Okumoto, C.H. Lee, A.J. Heeger, T. Ohnishi, and T. Noguchi, Phys. Rev. B 54, 4748 (1996).

${ }^{29}$ R.H. Friend, G.J. Denton, J.J.M. Halls, N.T. Harrison, A.B. Holmes, A. Köhler, A. Lux, S.C. Moratti, K. Pichler, N. Tessler, and K. Towns, Synth. Met. 84, 463 (1997).

${ }^{30}$ S. Frolov, M. Liess, P.A. Lane, W. Gellermann, Z.V. Vardeny, M. Ozaki, and K. Yoshino, Phys. Rev. Lett. 78, 4285 (1997).

${ }^{31}$ J.W. Blatchford, S.W. Jessen, L.B. Lin, J.J. Lih, T.L. Gustafson, A.J. Epstein, D.K. Fu, M.J. Marsella, T.M. Swager, A.G. MacDiarmid, S. Yamaguchi, and H. Hamaguchi, Phys. Rev. Lett. 76, 1513 (1996).

${ }^{32}$ M. Pope and C.E. Svenberg, Electronic Processes in Organic Crystals (Clarendon Press, Oxford, 1982).

${ }^{33}$ R.H. Austin, G.L. Baker, S. Etemad, and P. Thompson, J. Chem. Phys. 90, 11 (1989).

${ }^{34}$ M. Wohlgenannt, W. Graupner, R. Österbacka, G. Leising, D.
Comoretto, and Z.V. Vardeny, in ICSM 98 Proceedings, edited by P. Bernier, G. Bidan, and S. Lefrant (Elsevier, Amsterdam, in press).

${ }^{35}$ W.V. Houston, Phys. Rev. 57, 184 (1940).

${ }^{36}$ R. Österbacka, M. Wohlgenannt, D. Chinn, and Z.V. Vardeny, Phys. Rev. B (to be published).

${ }^{37}$ M. Rohlfing and S.G. Louie, Phys. Rev. Lett. 82, 1959 (1999).

${ }^{38}$ M. Chandross, S. Mazumdar, S. Jeglinski, X. Wei, Z.V. Vardeny, E.W. Kwock, and T.M. Miller, Phys. Rev. B 50, 14702 (1994).

${ }^{39}$ S. Barth, H. Bässler, U. Scherf, and K. Müllen, Chem. Phys. Lett. 288, 147 (1998).

${ }^{40}$ A. Köhler, D.A. Dos Santos, D. Beljonne, Z. Shuai, J-L. Brédas, A.B. Holmes, A. Kraus, K. Müllen, and R.H. Friend, Nature (London) 392, 903 (1998).

${ }^{41}$ M. Wohlgenannt, W. Graupner, G. Leising, and Z.V. Vardeny, Phys. Rev. Lett. 82, 3344 (1999).

${ }^{42}$ W. Graupner, G. Cerullo, G. Lanzani, M. Nisoli, E.J.W. List, G. Leising, and S. DeSilvestri, Phys. Rev. Lett. 81, 3259 (1998).

${ }^{43}$ Z. Vardeny and J. Tauc, in Semiconductors Probed by Ultrafast Laser Spectroscopy, edited by R.R. Alfano (Academic Press, New York, 1984), Vol. II, p. 23.

${ }^{44}$ V.I. Arkhipov, E.V. Emelianova, and H. Bässler, Phys. Rev. Lett. 82, 1321 (1999).

${ }^{45} \mathrm{~S}$. Mazumdar and M. Chandross, in Primary Photoexcitations in Conjugated Polymers; Molecular Excitons vs Semiconductor Band Model (Ref. 1), p. 384.

${ }^{46}$ I. H. Campbell, T. W. Hagler, D. L. Smith, and J. P. Ferraris, Phys. Rev. Lett. 76, 1900 (1996). 